\title{
Combining Image Simulation and Molecular Dynamics to Understand In-Situ Liquid Microscopy
}

\author{
D. A. Welch, 1 N.D. Browning, 2 J.E. Evans, 3 and R. Faller 1 \\ 1 \\ Department of Chemical Engineering and Materials Science, University of California Davis, One \\ Shields Avenue, Davis, CA, 95616, USA
}

2

Fundamental and Computational Sciences Directorate, Pacific Northwest National Laboratory P.O. Box 999, Richland, WA, 99352, USA

3

3 Environmental Molecular Sciences Laboratory, Pacific Northwest National Laboratory P.O. Box 999, Richland, WA, 99352, USA

Through environmental cell TEM, real-time observations are made of in situ chemical and biological processes with sub-nanometer spatial resolution [1,2]. Environmental cell design has seen many advancements in recent years that have resulted in excellent image contrast in the study of various systems, including macromolecular protein complexes, whole cells, nanoparticles, catalysts, and electrochemical systems [1-6]. For these in situ experiments, atomic resolution capabilities and image interpretation are intimately related to the exact experimental conditions used. It is thus necessary to develop our understanding of how the in situ environment along with other imaging conditions influence the obtained image. Image simulation can give this needed understanding, but in order to perform the image simulation, a partnership with molecular dynamics (MD) must be established in order to account for both the time-dependent structural evolution of specimens and the in situ environment itself $[7,8]$.

Structural descriptions of specimens as determined with MD enable the accuracy of image simulation techniques $[7,8]$. Whether the specimen is an inorganic or organic system, the time-dependent atomic movements of the specimen will influence image contrast. In the case of inorganic nanoparticles, as shown in Figure 1, MD can be used to calculate both thermal Debye-Waller factors and structural relaxation effects which are in turn used in the image simulation of the nanoparticle. MD also determines the structure of the in situ holder itself, allowing for a complete image simulation upon the entire in situ environment. Additionally, MD can determine structural variations of the specimen caused by the in situ environment such as those due to: 1) solvent stabilization of specimen structure 2) specimen-window interaction and 3) local momenta fluctuations. By understanding how all these factors contribute to the experimental image gathered over a given timescale, the capabilities of in situ electron microscopy can be predicted ahead of time before future experimental efforts.

In order to demonstrate in situ image simulation coupled with MD, we have shown that the experimental resolution of (220) $\mathrm{PbS}$ lattice fringes for nanoparticles grown in the fluid-stage can be reproduced with image simulation [1]. Clear relationships have been determined for the effects of window membrane thickness, fluid path length, defocus, and particle size on atomic resolution. In addition, we consider how the physical behavior of specimens within the fluid-stage holder may influence the time-dependent specimen structure. 


\section{References}

[1] Evans, J. E. Jungjohann, K. L. Browning, N. D. Arslan, I. Nano Lett. 11. 2011. 2809.

[2] Ring, E. A. de Jonge, N. Microsc. Microanal. 16. 2010. 622.

[3] Ross, F. M. In Situ Transmission Electron Microscopy. Hawkes, P. W. and Spence, J.C.H. (Eds.) Science of Microscopy. Springer Science+Business Media, LLC. New York. 445-534. 2007.

[4] Woehl, T. J. Evans, J. E. Arslan, I. Ristenpart, W. D. Browning, N. D. ACS Nano. 6. 2012. 8599.

[5] Evans, J. E. et al. Micron. 43. 2012. 1085.

[6] Parent, L. R et al. ACS Nano. 6. 2012. 3589.

[7] E. Kirkland. Image Simulation in Transmission Electron Microscopy. Cornell University. Ithaca. 2006.

[8] C. Koch. Determination of Core Structure Periodicity and Point Defect Density Along Dislocations. Dissertation. Arizona State University. 2002.

[9] A portion of this work was supported by the U.S. Department of Energy, Office of Basic Energy Sciences, Division of Materials Science and Engineering. Pacific Northwest National Laboratory (PNNL) is a multiprogram national laboratory operated for DOE by Battelle under Contract DE-AC05-76RL01830 and Grant DE-FG52-06NA26213. This work was also supported by the National Institute of Health (Grant RR025032-01 and Grant 5RC1GM091755.
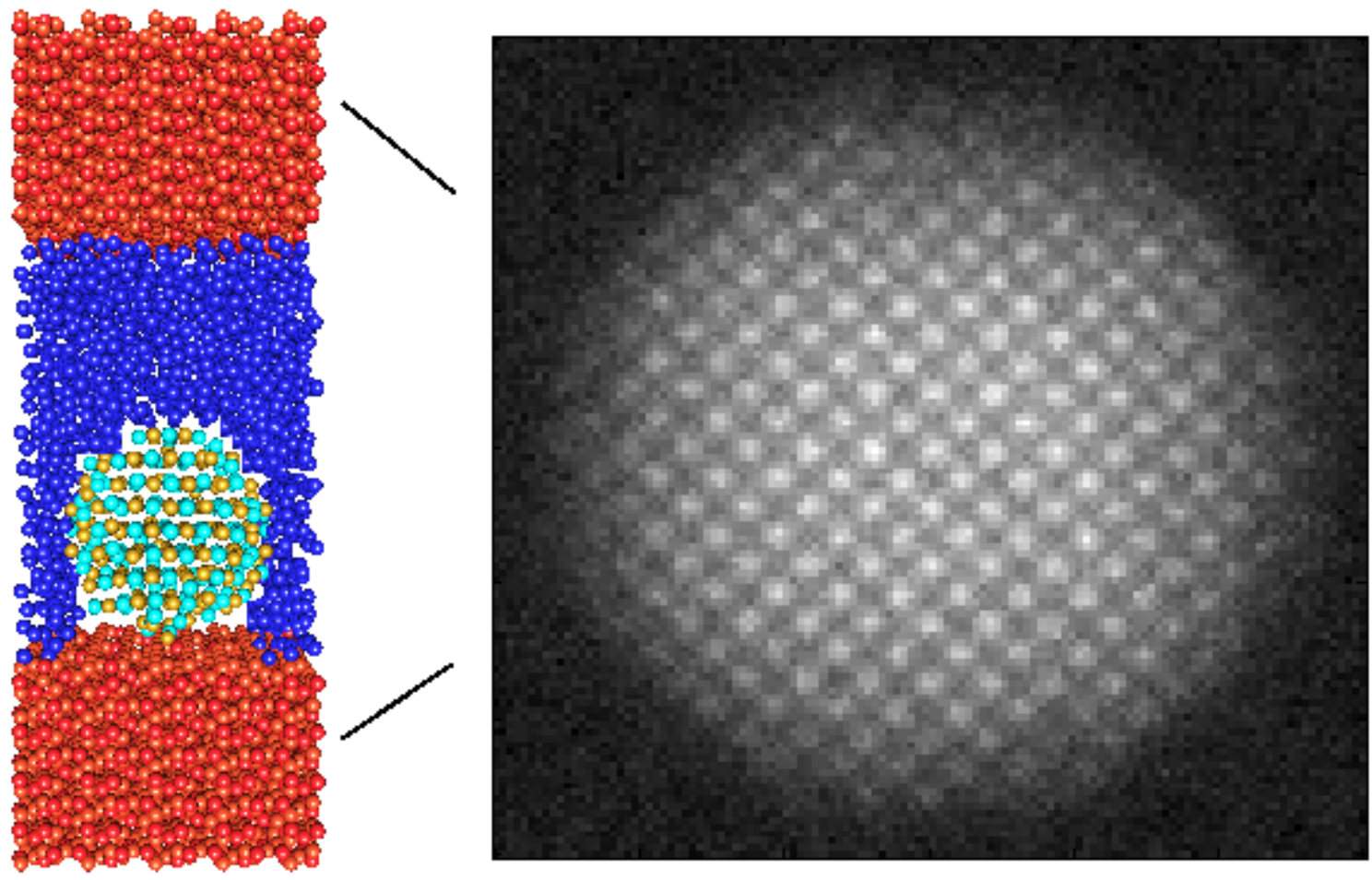

Figure 1. Structural modeling (left) and image simulation (right) of an inorganic nanoparticle in the in situ environment. MD simulation is used to determine the atomistic structure and thermal vibrations of a PbS nanoparticle specimen. In addition, structural descriptions of the in situ environment itself (i.e. the fluid and window membranes) is also determined. These findings are used as input for image simulation, resulting in a calculated image that predicts the contrast features specific to the in situ imaging conditions. 